\title{
LARVAL-SPECIFIC PROTEIN IN THE ORDER DICTYOPTERA-II. ANTAGONISTIC EFFECTS OF ECDYSONE AND REGENERATION ON LSP CONCENTRATION IN THE HEMOLYMPH OF THE ORIENTAL COCKROACH, BLATTA ORIENTALIS
}

\author{
JOSEPH G. KUNKEL \\ Zoology Department, University of Massachusetts at Amherst, Amherst, Massachusetts 01002, \\ U.S.A.
}

(Received 18 April 1974)

\begin{abstract}
Larval-specific protein, LSP, reaches a peak concentration in cockroach hemolymph 24$48 \mathrm{hr}$ prior to ecdysis.

2. When ecdysis is delayed by autotomy-induced leg regeneration, LSP continues to accumulate and reaches a higher than normal peak concentration $28 \mathrm{hr}$ prior to the delayed ecdysis.

3. Ecdysone inhibits the regeneration-induced delay and returns the normal pattern of ecdysis and LSP accumulation.

4. By using single and multiple autotomies it was demonstrated that the amount of accumulated LSP is proportional to the length of molting cycle delay rather than the amount of regenerating tissue.

5. It is suggested that the greater peak concentration of LSP seen in metamorphic molting cycles (Kunkel \& Lawler, 1974) is a function of the greater length of metamorphic molting cycles rather than being specifically a metamorphic phenomenon.
\end{abstract}

\section{INTRODUCTION}

A MAJOR larval-specific protein, LSP, has been demonstrated in the hemolymph of cockroaches and their closest relatives, the termites and preying mantids (Kunkel \& Lawler, 1974). Since the pattern of LSP concentration was found to be correlated with the molting cycle and influenced by metamorphosis, the present study was conceived to see how modulating the molting cycle by natural and artificial means would affect LSP concentration. Autotomy of a leg was used to delay the molting cycle (O'Farrel \& Stock, 1953). Ecdysone, which stimulates cuticle deposition by regenerating legs in vitro (Marks \& Leopold, 1970), was used to overcome the autotomy-induced delay of the molting cycle in vivo (Bulliere, 1972).

\section{MATERIALS AND METHODS}

Synchronous cultures of the Oriental cockroach, Blatta orientalis, were maintained at $30^{\circ} \mathrm{C}$ as described previously (Kunkel, 1966; Kunkel \& Lawler, 1974). Fifth instar larvae were chosen to study because they allowed a $5 \mu \mathrm{l}$ serum sample to be taken easily and also because this instar is the last instar in which no proportion of the individuals metamorphose to the adult (Kunkel, in preparation).

The relative concentration of LSP was measured immunologically by Oudin single diffusion as described previously (Kunkel \& Lawler, 1974).
Ecdysterone (Schwarz-Mann) was injected in $1 \mu 1$ of distilled water into the haemocoel of $\mathrm{CO}_{2}$ anaesthetized larvae with a $10 \mu 1$ Hamilton syringe.

\section{RESULTS}

Inhibition of regeneration delay by ecdysterone

Since excessive amounts of ecdysone can produce abnormal molting (Williams, 1968; Schneiderman, 1972), a dose of ecdysterone sufficient to inhibit regeneration and promote normal molting of a culture had to be estimated. To do this, a culture of cockroaches fed at time zero was anaesthetized at $96 \mathrm{hr}$ and a metathoracic leg was autotomized at the trochanter-femur autotomy plane. Notably at this autotomy time, 100 per cent of the animals would delay their molting cycle to regenerate a leg. One $\mathrm{hr}$ after autotomy, groups of twenty-five larvae were injected with graded doses of ecdysterone. The injected ecdysterone has an all or none effect on inhibiting the delay of molting. Figure 1 demonstrates the proportion of the injected animals in which regeneration was inhibited and molting occurred without a delay. A cumulative normal curve was fitted to the data by probit analysis (Finney, 1952). This curve allows one to predict the dose of ecdysterone necessary to achieve any desired percentage of inhibition. The dose of ecdysterone capable of causing 50 per cent inhibition of molting delay in fifth instar 


\section{JOSEPH G. KUNKEL}

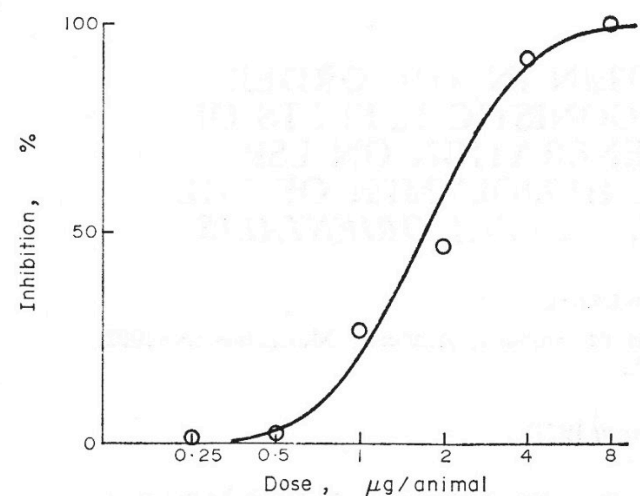

Fig. 1. Log dose response curve for ecdysterone's effect on inhibiting regeneration in B. orientalis. Fifth instar larvae had a leg removed $96 \mathrm{hr}$ after feeding, doses of ecdysterone were injected I hr later. The proportion of twenty-five animals in which regeneration and delay of molting were inhibited is plotted against the concentration injected (1092 scale). A cumulative normal curve was fitted to the data by probit analysis

larvae is $1.7 \mu \mathrm{g}$. In order to ensure a 95 per cent inhibition of delay a dose of $5.3 \mu \mathrm{g}$ is predicted. However, the actually injected dose of $8.0 \mu \mathrm{g}$, Fig. 1, while causing 100 per cent inhibition of delay, caused larvae to reach ecdysis much earlier than unautotomized controls, and 60 per cent of the larvae were unable to free themselves from their old cuticle. A dose of $5 \mu \mathrm{g}$ was chosen as the best compromise between maximal inhibition of delay and minimal abnormality of molting. This dose in subsequent tests was shown to result in close to 100 per cent inhibition of delay while causing no obvious abnormalities of molting.

Effect of ecdysone and regeneration on LSP concentration

A culture of 250 fifth instar larvae were fed at time zero. A $5 \mu 1$ sample of hemolymph was taken for LSP analysis from five animals randomly selected from the culture at 24-hr intervals. Animals were discarded after being bled. At $96 \mathrm{hr}$ the culture was divided into three treatment groups. One group was retained as an autotomized control, A second group had a metathoracic leg autotomized at the trochanter under $\mathrm{CO}_{2}$ anaesthesia. The third group had a leg similarly autotomized and $590 \mu \mathrm{g}$ of ecdysterone was injected into them I hr later. Subsequently, samples of five animals were taken from each treatment group at 24-hr intervals and $5 \mu \mathrm{l}$ of hemolymph was drawn from each for LSP analysis. Figure 2 shows the changes of LSP which occur in response to the three experimental treatments. During the delay caused by regeneration in the autotomized group, LSP continues to accumulate at a slow rate, When ecdysis in this group finally occurs, the LSP has reached a concentration much higher than

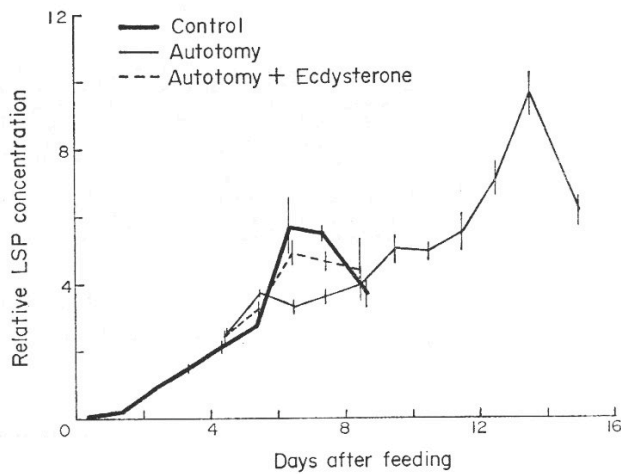

Fig. 2. Effect of regeneration and ecdysterone on LSP accumulation during the $5^{\text {th }}$ instar of B. orientalis. ( Control animals unautotomized and uninjected; $(-)$ autotomized $96 \mathrm{hr}$ after feeding; (-----) autotomized $96 \mathrm{hr}$ after feeding and injected with 5 jig of ecdysterone. The vertical bars represent 1 S.E. about the mean LSP concentration for five animals. The last point in each curve marks the LSP concentration at ecdysis.

the unautotomized controls. The ecdysone-injected group did not delay its molt to regenerate the autotomized limb and under the influence of the $5 \mu \mathrm{g}$ of ecdysterone molted slightly ahead of the controls. This slightly premature molting may be causally related to the slightly lower levels of LSP which were measured for the ecdysterone-injected group. All three groups show a peak of LSP 1-2 days before ecdysis.

\section{Effects of simultaneous vs sequential regeneration on LSP concentration}

The accumulation of more LSP in regenerating animals could be attributed directly to the regenerating tissue. In order to examine this possibility, animals were subjected to various amounts and duration of regeneration tissue. It has been previously reported (Stock \& O'Farrell, 1954), that taking off two legs simultaneously causes a slight extra delay compared to taking off one leg. Also it had been shown that taking off legs sequentially at intervals would delay the molting cycle considerably longer than single leg autotomy (O'Farrell \& Stock, 1954)

A culture of 300 fifth instar animals were fed at time zero and at $96 \mathrm{hr}$ after feeding they were divided into 3 groups of 100. One group had one meta-thoracic leg autotomized at $96 \mathrm{hr}$, at the trochanter-femur autotomy point, and another group had two metathoracic legs autotomized, both at $96 \mathrm{hr}$. The third group had two legs autotomized sequentially, one at $96 \mathrm{hr}$ and one at $216 \mathrm{hr}$ after feeding. Samples of hemolymph were taken at 24-hr intervals until $24 \mathrm{hr}$ after ecdysis in each treatment group. Figure 3 shows that there is no apparent difference in LSP accumulation caused by one vs. two legs regenerating as long as the autotomies occurred simultaneously. However, when additional 


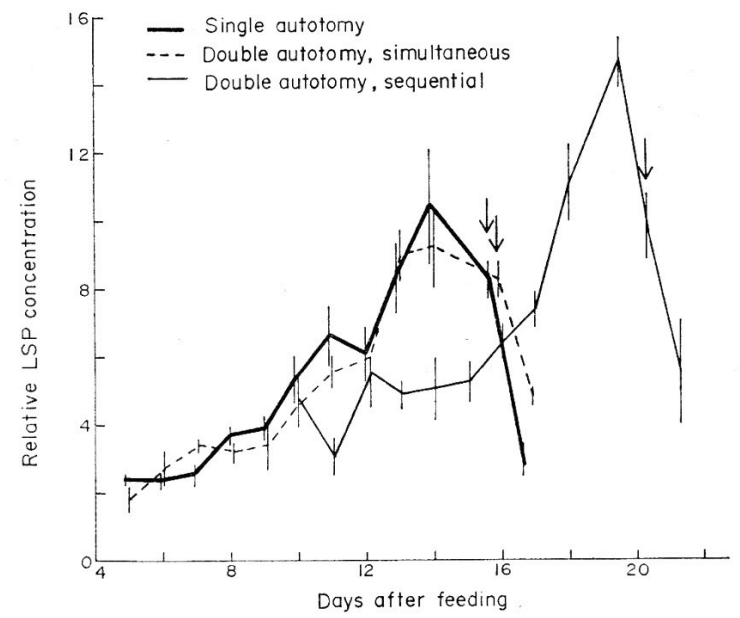

Fig. 3. Effect of various degrees of regeneration on LSP accumulation during the fifth instar of B. orientalis. ( $\longrightarrow)$ Single autotomy at $96 \mathrm{hr}$ after feeding; (- simultaneous double autotomy at $96 \mathrm{hr}$; (-----) sequential double autotomy, one leg at $96 \mathrm{hr}$, one leg at $216 \mathrm{hr}$. The vertical bars represent 1 S.E. about the mean LSP concentration for five animals. The last point in each curve represents the LSP concentration $24 \mathrm{hr}$ after ecdysis. LSP concentration at ecdysis for each group is indicated by an arrow.

delay is caused by taking off a second leg $120 \mathrm{hr}$ after a first leg, additional LSP accumulates. These results demonstrate that the accumulation of LSP is related to the length of the delay of molting and not the amount of regenerating tissue,

\section{DISCUSSION}

A pattern of LSP concentration change related to ecdysis has been demonstrated. Two rates of accumulation are observed*. one slow rate, whch starts shortly after feeding, and another faster rate starting about 3 days prior to ecdysis. LSP concentration reaches a peak 1-2 days prior to ecdysis whether the ecdysis occurs normally, is delayed by leg regeneration or is induced by injecting ecdysone. Although a stereotyped pattern of concentration change seems to be locked to the time of ecdysis, the amount of LSP accumulated at peak concentration varies considerably. The longer the molting phase is delayed by regeneration, the more LSP accumulates at the slow rate and the higher the eventual peak concentration prior to ecdysis. A similar phenomenon was observed previously in comparing the metamorphic molting cycle to larva-to-larva molting cycles (Kunkel \& Lawler, 1974): The metamorphic molting cycle in cockroaches is considerably longer than non-metamorphic molting cycles. Three successive larva-to-larva molting cycles (IV-VI) were shown to have indistinguishable LSP concentration patterns, but during the longer metamorphic cycle (VII) LSP reached a much higher concentration. It seems, therefore, based on the results of the above experiments that the higher peak concentration of LSP observed during the metamorphic molt is not strictly a metamorphic phenomenon but is more likely related to the longer instar length entailed by the metamorphic process.

Why LSP should continue to accumulate in regeneration-delayed animals is obscure. It may be related to the compensatory shortening of subsequent stadia observed by O'Farrell \& Stock (1953).

Acknowledgements-This research was supported by a faculty research grant FR-J39-72 from the Research Council of the University of Massachusetts at Amherst. I am indebted to Gerda I. Kunkel for technical assistance.

\section{REFERENCES}

Bulliere D. (1972) Action de 1'ecdysone et de l'inokosterone sur la régénération d'appendice chez la larva de Blabera cranifer (Insect, Dictyoptère). C.r. hebd. Séanc. Acad. Sci., Paris D 274, 1349-1352.

FinNey D. J. (1952) Probit Analysis: A Statistical Treatment of the Sigmoid Response Curve, 2nd edn. Cambridge University Press, London.

KUNKEL J. G. (1966) Development and availability of food in the German cockroach, Blattella germanica (L.). J. Insect Physiol. 12, 227-235.

KUNKEL J. G. \& LAWLER D. M. (1974) Larval-specific serum protein in the order Dictyoptera - I. Immunological characterization in larval Blattella germanica and cross-reaction throughout the order. Comp. Biochem. Physiol. 47B, 697-710 


\section{JOSEPH G. KUNKEL}

Marks E. P. \& LEOPOLD R. A. (1970) Cockroach leg regeneration, effects of ecdysterone in vitro. Science, Wash, 167, 61-62.

O'FArrell A. F. \& Stock A. (1953) Regeneration and the molting cycle in Blattella germanica (L.) - I. Single regeneration in the first instar. Aust. J. Biol. Sci. 6, 485-500,

O'FARRELl A. F. \& STOCK A. (1954) Regeneration and the molting cycle in Blattella germanica (L.) - III. Successive regeneration of both metathoracic legs. Aust, J. Biol. Sci. 7. 525-536.

SCHNEIDERMAN H. A. (1972) Insect hormones and insect control. In Insect Juvenile Hormones (Edited by Menn J. J. \& Beroza M.), pp. 327. Academic Press, New York,
Stock A. \& O'FArrell A. F. (1954) Regeneration and the molting cycle in Blattella germanica (L.). Simultaneous regeneration of both metathoracic legs. Aust. J. Biol. Sci. 7. 302-307.

Williams C. M. (1968) Ecdysone and ecdysone analogues: their assay and action on diapausing pupae of the cynthia silkworm Biol. Bull. Mar. boil. Lab., Woods Hole 134, 344-355o

Key Word Index- Insect; cockroach; serum protein; antigen; molting; metamorphosis; regeneration; ecdysone; Blatta orientalis. 